\title{
Revisión de la influencia de la motivación docente en el empleo de las pizarras digitales interactivas
}

\section{Review of the Influence of Teacher Motivation on the Use of Interactive Whiteboards}

\author{
Miriam M. Rojas-Segovia* \\ Universidad Tecnológica del Perú, Lima, Perú \\ Universidad Nacional Mayor de San Marcos, Lima, Perú \\ ORCID: https://orcid.org/0000-0001-5266-9548 \\ Douglas Y. Romero-Varela \\ Universidad Tecnológica del Perú, Lima, Perú. \\ ORCID: https://orcid.org/0000-0003-2158-9558
}

Recibido 18-07-18 Revisado 08-08-18 Aprobado 21-11-18 En línea 12-03-19

*Correspondencia

Email: mercedes.segovia149@gmail.com
Citar como:

Rojas-Segovia, M., \& Romero-Varela, D. (2019). Revisión de la influencia de la motivación docente en el empleo de las pizarras digitales interactivas. Propósitos $y$ Representaciones, 7(2), 516-535. doi: http://dx.doi.org/10.20511/pyr2019.v7n2.228 


\section{Resumen}

El presente artículo corresponde a la revisión de un tema que pretende otorgar una visión general acerca de los datos recabados sobre el uso de las pizarras digitales interactivas en el desarrollo de las sesiones de enseñanza-aprendizaje que toman como factor determinante la motivación de los docentes. Se realizó una examinación de la literatura disponible en línea para elaborar un análisis de la situación actual de esta innovadora herramienta didáctica y su relación con la predisposición del pedagogo que la emplea, como parte del proyecto final para la asignatura de Investigación Académica en la Universidad Tecnológica del Perú. Luego de la investigación, se llegó a la conclusión de que, a la fecha de edición, no existen suficientes investigaciones que centren su atención en la actitud del profesor, sobre todo en el Perú, por lo que no se puede determinar a cabalidad que exista una relación directa entre ambos aspectos y se incentiva a desarrollar proyectos que consideren la relevancia del educador en el empleo eficiente de la tecnología con el fin de lograr una educación de calidad y adaptada a los estándares de la sociedad de la información emergente.

Palabras clave: Educación y tecnología; Influencia del profesor; Motivación; TIC, Recursos tecnológicos.

\section{Summary}

The present article is a topic's revision and it pretends to give a general vision of the recollected data about the use of interactive digital boards in the sessions of teaching-learning process' development that takes as a determinant factor the teacher's motivation. It has been made an examination of the available literature online to elaborate an analysis of the actual situation of this innovating didactic tool and its relation with the teacher's predisposition, as part of the final project for the Academic Investigation subject in the Technological University of Peru. After the investigation, it concluded, until the edition's date, there are no enough researches that center their attention in the teacher's attitude, especially in Peru, so it can't be determined if there is a direct relation between these aspects and it's recommended to develop projects that gives relevance to the educator in the efficient use of the technology with the purpose of achieve a qualified education that is adapted to the emergent information society's standards.

Keywords: Education and Technology; Teacher's Influence; Motivation; TIC; Technological Resources.

\section{Introducción}

En los últimos años, se ha generado una necesidad inminente de acceso a la tecnología en todos los sentidos, de la cual se obtiene la información necesaria para desarrollar diversas actividades, específicamente, educativas. Ante dicho escenario, se han apreciado muchas iniciativas de mejora por parte de los docentes, quienes han presentado interesantes propuestas de implementación para facilitar tal proceso, estas metodologías integran, oportunamente, el uso y aplicación de herramientas tecnológicas que acerquen a los alumnos y alumnas a la data global existente para la optimización de sus capacidades y la práctica educativa cotidiana. Esto se dilucida en el contemporáneo trabajo que Prensky (2001) presentó, en el cual sentencia que la nueva generación "ha nacido y se ha formado utilizando la particular "lengua digital" de juegos por ordenador, vídeo o Internet" (p. 5), esta nueva sociedad tiene a menudo "la sensación de que a las aulas ha llegado, para instruirles, un nutrido contingente de extranjeros que hablan idiomas desconocidos, extranjeros con muy buena voluntad, sí, pero ininteligibles" (p. 6); es por esto que resulta inminentemente necesaria la actualización de este personaje dentro de la rutina educativa. Esta demanda implica estratégicamente las cualidades ligeramente exclusivas de la sociedad del Internet; quienes se caracterizan por su deseo inequívoco de "recibir la información de forma ágil e inmediata y de instruirse de forma lúdica a embarcarse en el rigor del trabajo tradicional" (Prensky, 2001, p.6); así como de su interesante atracción "por multitareas y procesos paralelos, 
por los gráficos más que los textos y por los accesos al azar (desde hipertextos)", ya que funcionan mejor y rinden más cuando trabajan en línea bajo la "conciencia de que van progresando, lo cual les reporta satisfacción y recompensa inmediatas" (p. 6) que puede extenderse a docentes tanto como a los estudiantes.

Es por ello que se ha percibido la creciente curiosidad acerca del uso, aplicación, beneficios y desventajas de las Tecnologías de Información y Comunicación (TIC). Esto se ha dado de tal manera, como consecuencia de la convergencia de tecnologías y mercados y de los avances producidos de la globalización económica y política. Ante dicho panorama, se ha evidenciado que "la tecnología no es un recurso que se utilice frecuentemente, sino más bien un artículo que está guardado en bodegas o en salas de poco uso" (Peña, 2014, p. 10); en comparación con otras TIC, para Duan (2010) "la singularidad y el interés de la PDI se encuentra en la posibilidad de conseguir una intersección entre la interactividad técnica y pedagógica" (p.144), aunque si bien existen estudios que analizan la actitud de los profesores hacia el uso de las TIC, estos tienen como objetivo identificar dicha actitud, determinar sus causas y/o categorizarla, sin analizar sus efectos. Es decir, pocos incluyen la predisposición de los docentes hacia su uso como uno de los múltiples factores que pueden determinar su pertinente incorporación al aula.

Es por ello que, se plantea un debate sostenido acerca de la posibilidad empírica de establecer una relación directa en la interacción entre la motivación docente y el pertinente uso de la tecnología, específicamente la Pizarra Digital Interactiva (PDI), pues algunas investigaciones señalan que la predisposición positiva o negativa del pedagogo ejerce un rol crucial en su práctica docente, consecuentemente, se observaría si los estudiantes adoptan correcta o equívocamente aquello que su mentor desea transmitir mediante el buen o mal uso de tal herramienta.

La idea de intervenir en el resultado positivo o negativo de la práctica docente sobre sus estudiantes se encuentra estrechamente relacionada con el concepto de influencia. Dahl (1985) define este término como "una relación entre actores, en la que uno de ellos induce a los otros a actuar de un modo en el que no lo harían de otra manera" (pp.103-104). Entonces, en el ámbito pedagógico, se comprende como la estrecha conexión entre los agentes educativos, en la que los docentes incurren en acciones que precisan a los estudiantes a proceder de cierta forma específica según los logros que se desean alcanzar.

Mediante el presente trabajo, se realiza una revisión bibliográfica acerca de las investigaciones sobre el tema desarrolladas en la última década, debido a que no se hallaron trabajos realizados en el Perú sobre este tópico, se consideran aquellos que se elaboraron a nivel internacional para otorgar un enfoque holístico e incentivar la aplicación de los conocimientos adquiridos en otros países y aplicarlos, previa adaptación, a la realidad peruana; para ello se revisan los factores relacionados a las facilidades e impedimentos que se han observado en ellas para motivar futuros estudios, con el fin de lograr la optimización del empleo de dicha herramienta en beneficio de la adaptación a las nuevas demandas de una sociedad del conocimiento bajo un enfoque tecnológico en la educación actual.

\section{Conceptos previos}

\section{Motivación docente.}

Una de las expresiones necesarias para comprender el presente trabajo es también la motivación docente, este concepto posee diversas implicancias, sin embargo, al ser mayoritariamente empíricas resulta muy complejo definirlo sin caer en el juicio valorativo de lo que aborda. A pesar de tal dificultad, se expresan algunas de las concepciones que se han realizado en diversas investigaciones sobre la motivación en general, Reeve (2012) explicita que la motivación comprende todo aquello que atribuye energía y dirección al comportamiento. 
En el mismo sentido, Ryan y Deci (2000) la describen como aquello que guía una acción, y de ello se deduce su crucial rol en la formación de personas a cargo de movilizar a otros en la ejecución de una actividad como los profesores, líderes o padres. Bajo el enfoque educativo, se considera el papel de los docentes como modeladores o instructores; ante ello Niemiec y Ryan (2009) refieren que la motivación "resulta relevante en el campo de la educación, en donde el interés natural del estudiante por aprender representa un gran recurso para el educador para fomentar el aprendizaje" (p. 134); por lo que Luna (2006) sugiere que tal concepto implica "pensar que el maestro ha de realizar en la enseñanza una serie de actividades que muevan emocional e intelectualmente al alumno hacia la realización de actividades de aprendizaje, a la obtención de los productos de éste y sus consecuencias futuras" (p.29).

De todo lo anterior, se infiere que este concepto es una característica que el pedagogo adquiere para mantener activamente una práctica educativa de calidad y que se transmite a los estudiantes para alcanzar los logros esperados respecto de su formación cognitiva, psicológica y actitudinal.

\section{Pizarra digital interactiva (PDI).}

En el rubro educativo, existen muchas herramientas didácticas que facilitan y optimizan la práctica docente, estas pueden ser estructuradas o no estructuradas, lo cual se refiere a que si se elaboran en serie y con las mismas características o son hechas por los propios docentes adaptándolas a la realidad educativa que perciben. Con el paso del tiempo, se han renovado estos recursos por otros que resulten más sencillos de transportar o que se ubiquen perennemente en el aula de clases y estén disponibles en cualquier momento para ser usados. La opción más pertinente y acorde con el boom del internet son los dispositivos tecnológicos, tales como computadoras, tabletas, celulares, proyectores multimedia, entre otros.

Con base en estos elementos, se han diseñado instrumentos que vinculen o integren más de uno de los mencionados anteriormente; así pues, se logró desarrollar un material estructurado, que es a la vez un conjunto de herramientas tecnológicas, que pueda ser implementada en las aulas de clase para reemplazar las tradicionales pizarras a tiza o acrílicas por las PDI. Según Corrales (2015) "la PDI se puede definir como una herramienta tecnológica, básicamente compuesta por una pantalla sensible de diferentes dimensiones, un ordenador multimedia y un proyector" (p. 161); de manera similar, para Murado (2012) "se trata de un sistema compuesto por un ordenador y un proyector de vídeo que permite mostrar sobre una superficie una serie de contenidos en formato digital" (párr. 8). Así pues, se entiende por PDI, un sistema que integra una computadora, un proyector multimedia y una pantalla sensible en la que se puede anotar utilizando los dedos o con un puntero especial que presenta un contenido que puede ser manipulado desde el ordenador, con el fin de acercar a los estudiantes a interactuar con el mundo.

\section{Tecnologías de la Información y Comunicación (TIC).}

Este concepto refiere a un conocimiento adquirido recientemente acerca del desarrollo informático masivo que se ha experimentado en la última década. Es por ello, que para enmarcar la variable definida en el apartado anterior en una clasificación general, aún más general que la de recursos educativos, se ubica a las PDI y las demás herramientas con estructuración y fines comunes, en el marco de las TIC. Estas son definidas por el Programa de las Naciones Unidas para el Desarrollo (PNUD, 2002), citado por Daccach (s.f) como:

El universo de dos conjuntos, representados por las tradicionales Tecnologías de la Comunicación (TC) -constituidas principalmente por la radio, la televisión y la telefonía convencional- y por las Tecnologías de la Información (TI) caracterizadas por la digitalización de las tecnologías de registros de contenidos (informática, de las comunicaciones, telemática y de las interfaces) (p. 1). 
La Sociedad de Información de Telefónica, como se cita en Sánchez (2008), define las TIC como: "tecnologías que se necesitan para la gestión y transformación de la información y muy en particular, el uso de ordenadores y programas que permiten crear, modificar, almacenar, proteger y recuperar esa información" (p. 156). A través de estas percepciones, se sintetiza a las TIC como un medio de continuidad y transmisión de la información disponible en la sociedad del conocimiento para garantizar su exposición al resto de ciudadanos de la comunidad científica y permitir el desarrollo de aún más contenidos.

\section{Educación basada en las TIC.}

Tras explicar los conceptos de motivación docente y las TIC, resulta oportuno destacar un proceso que las vincula estrecha y concretamente en resultados específicos motivados por todos los agentes implicados; es decir, se considera en este punto el concepto de una formación bajo un enfoque de aplicación de las TIC para desarrollar las competencias inherentes a esta visión tecnológica. Para ello, es preciso tomar en cuenta aquello que resalta Martín (2009) al asegurar que:

El cambio del rol del profesor, de depositario a guía del conocimiento; el principio de aprender a aprender como columna vertebral del modelo cognitivo; la interacción de lo individual y lo grupal, pilares que sustentan esta visión del aprendizaje, se ensamblan de manera particularmente idónea con los usos y modelos que ofrecen las TIC y, de forma especial, Internet. (p. 55).

De ello, se desliga que el papel del profesor es crucial en la implementación de dispositivos tecnológicos que agilicen su desempeño en las aulas. Es importante comprender el modelo de aprendizaje que implica la incorporación de las TIC, Gatica y Valdivia (2014) la definen como aquella que "permite a los educandos participar activamente de un aprendizaje colaborativo en donde la creación de instancias que promueven el compartir objetos de aprendizaje contribuyen a enriquecer significativamente el proceso educativo" (p. 203).

En concordancia con lo referido, se continúa enfatizando en el docente como esencial motor de cambio para instaurar este enfoque educativo, que implica necesariamente, la necesidad de inculcar en los estudiantes la autonomía en su aprendizaje, así como evidenciar la exigencia de un cambio en la administración y concepción de la educación "en la dirección de formar personas que gestionen sus propios aprendizajes, adopten una autonomía creciente y dispongan de herramientas intelectuales que les permitan un aprendizaje continuo a lo largo de la vida" (Díaz, 2009, p. 139) y lograr tan ansiada meta.

\section{Argumentación.}

\section{Clima del aula y las PDI.}

Una tesis doctoral presentada en España, planteó el desarrollo de 6 sesiones de aprendizaje en un aula de 27 estudiantes para la clase de música con el uso de una pizarra digital interactiva, para comprobar la influencia de esta herramienta sobre el clima del aula; por ello Monreal (2013) detalla que "no solo la formación es importante, también lo es, de manera determinante, la mentalidad del profesor, su carácter flexible y su capacidad para implementar la cultura de riesgo en el aula" (p. 224). La metodología empleada consta de la aplicación directa de instrumentos para la recolección de datos, tales como la elaboración de entrevistas formales e informales, cuestionarios, diarios de campo, la observación explícita, entre otros. Tras la experimentación, se evidenciaron diversas dificultades, tales como las deficiencias técnicas de esta herramienta durante las clases, pero se resaltan las mejoras en el ambiente tales como el cambio de actitud tanto de estudiantes como del maestro; pues como lo explica la autora: 
Es importante tener presente que la verdadera innovación metodológica parte primero del profesor; debe ser el propulsor de dicho cambio, pues sin su voluntariedad y sin su actitud abierta y reflexiva hacia otra metodología en la manera de enseñar, nunca se podrá contar con una verdadera innovación metodológica que se acerque a la realidad social. (pp. 36-37).

Se seleccionaron ocho macrocategorías que abordaron los criterios a evaluar con base en las dimensiones específicas de las variables de estudio; cada una de ellas estaba constituida por preguntas que permitieron otorgar un juicio determinado en la calificación 232final de cada aspecto. Esencialmente, se describe como una investigación de cráter cualitativo y basado en un estudio de casos único que concluyó con la relativa negación del planteamiento inicial, debido a que no se realizó una integración curricular coherente y eficiente de la PDI como recurso didáctico; "esto puede ser debido, por un lado a la falta de creatividad del profesor, y por otro, por la falta de tiempo para generar recursos didácticos adaptados" (Monreal, 2013, p. 232). Ambas razones convergen de la deficiente motivación que demuestra el docente, pues en concordancia con lo que explican Gatica y Valdivia (2014) "la pizarra digital interactiva fomenta la flexibilidad y la espontaneidad de los docentes" (p. 200), lo cual no se demostró en el caso de la escuela elegida para esta investigación.

\section{Uso de las TIC en la práctica docente.}

El grupo de investigación DIM-UAB, con el patrocinio de EPSON emprendió el proyecto "centros piloto Epson" que consistió en proporcionar apoyo didáctico y económico a 15 centros docentes de la etapa de enseñanza obligatoria y bachillerato para que acaben de completar sus dotaciones y puedan disponer de una pizarra digital en cada una de sus aulas de clase, con el fin de aprovechar al máximo sus posibilidades didácticas. Durante los años 2006 y 2007, este estudio proyectado a la identificación de buenas prácticas docentes recolectó información para su posterior difusión a otros centros y así conseguir mejores experiencias de aprendizaje basado en el uso y aplicación de las TIC. La metodología aplicada consistía en solicitar, antes de iniciar el proyecto, que los 220 profesores, a lo largo de la investigación, participaran en los cuatro seminarios de formación que contarían con un tutor que instruiría en el área tecnológica y didáctica; y que rellenen, en su momento, los informes correspondientes. Se aplicaron diversos cuestionarios y varias entrevistas personales bajo una constante supervisión por parte de los tutores asignados para recabar los resultados del trabajo educativo.

Al finalizar la investigación, los docentes alegaron que existen más ventajas que desventajas al emplear las TIC en sus clases; también manifestaron que aprender a usarlas es fundamental en la sociedad actual, así como la relativa sencillez de elaboración de material por su carácter digital. Todo esto, implicaría que aprender y utilizar las nuevas herramientas TIC, tan importantes en la sociedad actual, así como la renovación de las metodologías docentes con la aplicación de estos modelos, aumenta la satisfacción, motivación y autoestima docente, siempre que se considere prioritario su utilización en la propia aula y no en un salón separado de uso común con otras clases.

\section{Los docentes opinan sobre las PDI.}

Una tesis elaborada en Costa Rica, de tipo cualitativa con un enfoque naturista e interpretativo, buscó determinar el impacto que poseen las PDI en las aulas de una escuela primaria, con énfasis en los beneficios que podrían presentar en estudiantes y docentes a través de un proceso inductivo con base en un estudio de casos, la muestra seleccionada corresponde al tercer grado mediante la comparación de las clases dadas con y sin empleo de la PDI en todas las materias básicas del currículo. Se realizaron entrevistas personalizadas para explayar acerca de las categorías elegidas a evaluar, dentro del apartado de "opiniones del docente" se consideraron la percepción y disposición o actitud, ambas comparadas también con la observación dirigida. Al revisar los resultados, "se identificó que el sentimiento docente es positivo en todas las direcciones; hacia la herramienta, hacia la necesidad de capacitación en su utilización apropiada y la confianza de que 
es una herramienta útil y beneficiosa" (Díaz, 2012, p. 82); ello se concluyó con referencia a los criterios de evaluación elegidos, enmarcados en la "frecuencia de uso, el sentimiento personal sobre la herramienta y la disposición para utilizarla" (Díaz, 2012, p. 68), finalizando con la idea de que la PDI representa una herramienta eficiente para su labor educativa y que los docentes de dicha muestra demostraron satisfacción al emplearla a pesar de que no creen tener el tiempo que quisieran para explotar sus funcionalidades; ya que como Gónzalez (2018) menciona, "el objetivo de la pizarra digital interactiva en el ámbito educativo es la de facilitar al docente el acceso a diversos contenidos de índole técnico pedagógico y así presentar a los alumnos la oportunidad de interactuar con el mundo" (p. 89)

Se debe recalcar que "la materia impartida durante la aplicación no se considera una limitante significativa porque la investigación no tenía relación con el contenido de la lecciones sino más bien se enfocó a describir las actitudes y desempeños de los sujetos involucrados" (Díaz, 2014, p. 85); y que respecto a los instrumentos utilizados para recolectar datos, las entrevistas a los docentes, a los estudiantes y a los grupos de estudiantes, y las observaciones, no existió ningún obstáculo que debiera superarse para su aplicación; por todo esto se recomendó "estudiar la actitud y disposición de los docentes hacia la PDI y hacia la necesidad de capacitarse en su utilización ya que de eso dependen los beneficios que se obtengan del uso de la herramienta" (Díaz, 2014, p. 87).

\section{Docentes motivados, estudiantes motivados.}

Una investigación realizada en 20 escuelas de España durante el período de 2008 al 2010, pretendía describir los usos de las PDI más empleados, así como sus ventajas, inconvenientes, entre otros. Se empleó una metodología similar a correspondiente en la investigación-acción, además se proyectaron dos "acciones formativas", que se explican de la siguiente manera:

Una acción formativa (en cada curso) es un congreso con sesiones formativas pedagógicas y sobre técnicas básicas de la PDI, foros de preguntas y respuestas, y orientaciones específicas. La otra acción formativa (en cada curso) es un seminario en cada uno de los centros donde los profesores comentan lo que están realizando, y el coordinador orienta y proporciona nueva formación (p. 103).

Se aplicaron fichas de actividades monitorizados, cuestionarios e informes o actas de seguimiento extraídos de la observación, participación e integración en los congresos ofrecidos. En cantidades porcentuales, se halló que "prácticamente todo el profesorado utiliza la PDI en más del $60 \%$ de las clases y la mitad del profesorado la emplea en más del $80 \%$ de su actividad docente" (Domingo, 2011, p. 108). Eficazmente, lo más importante para el tratado del presente artículo, se halló que "el profesorado destaca la renovación metodológica y el aumento de satisfacción, motivación y autoestima" (Domingo, 2011, p. 113) en ellos mismos y en sus estudiantes, luego de implementar correctamente las PDI; lo cual se refuerza en el hecho de que "obviamente la eclosión de las redes digitales de todo tipo, la socialización que impulsa Internet, facilita el fortalecimiento de la componente grupal social, propiciando un espacio de encuentro efectivo y positivo entre la practica pedagógica y los avances tecnológicos" (Martín, 2009, p. 81).

\section{Predisposición docente y nivel de logro.}

El trabajo de Peña (2014) se realizó "sobre los datos obtenidos en la aplicación del curso de formación para el uso de Pizarra Digital Interactiva en el colegio San Sebastián de Quilicura acreditado por SENCE código N: 1237910395 a 59 docentes divididos en 2 grupos" (p. 26). Esta tesis fue elaborada para determinar que mientras más positiva sea la predisposición de los docentes, su desempeño didáctico mejora; ya que "la implicación del profesor que acompañe, filtre, organice y oriente el aprendizaje y que se siente arropado con el conocimiento social; una infraestructura tecnológica suficiente y adaptada a cada situación particular" (Martín, 2009, p. 58) es fundamental para el desempeño pedagógico. 
El autor consideró tres variables para su posterior análisis, las dos primeras son las mismas que se enfatizan a lo largo del presente documento, y también tomó el nivel de logro que los docentes demuestren al final de un curso de formación tecnológica acerca de las PDI como tercera implicancia, el cual fue medido a través de dos evaluaciones que se aplicaron al inicio y al final del mismo. Los resultados obtenidos en esta investigación mostraron que poco más de la mitad de los docentes evaluados presentaba una predisposición positiva frente al uso de las PDI; también se halló que la mayoría del total de profesores que pertenecen a la muestra, alcanzaron un nivel de logro avanzado en el uso de esta herramienta. Luego de procesar los datos estadísticos recabados de la experimentación propuesta, se determinó que la correlación entre los últimos criterios mencionados es muy baja según el estándar de Pearson; después de este hallazgo se procedió a realizar un análisis de varianza (Anova), sin embargo, tampoco se obtuvo significancia alguna, puesto que dicho trabajo no supone una concordancia estadística importante en cuanto a los cálculos realizados, debido a la baja confiabilidad empírica que ofrecen los instrumentos utilizados para la recolección de datos en la muestra seleccionada. Ello motivó al autor a incentivar a los receptores de su investigación a continuar trabajando en la materia, elaborar instrumentos válidos, seleccionar muestras más amplias y desarrollar estudios similares en otras áreas relevantes en la sociedad.

\section{Conclusiones}

A lo largo de los años, gracias a diversas investigaciones, se han desarrollado proyectos entusiastas orientados a la indagación de las ventajas e inconvenientes que pueda ofrecer la PDI en el aula de clases, ya sea para enseñar un tópico específica de una asignatura en particular o para todas ellas; así como se ha pretendido demostrar que dicho instrumento logra incrementar la atención y concentración de los estudiantes, tanto como su participación y mejora académica. Sin embargo, son pocas las investigaciones realizadas con el foco de atención en el docente, en el rol que cumple y sobre todo, en su motivación; es por ello que al iniciar esta revisión, se creía hallar numerosos trabajos sobre el tema, pero no fue así y para tornar el escenario más sombrío, fueron más escasos los trabajos realizados a nivel nacional.

Esto último conlleva a más preguntas que respuestas, si en el ámbito internacional, aunque en medidas regulares, se ha demostrado la eficacia de las PDI en el proceso de enseñanzaaprendizaje, ¿por qué no se trabaja para averiguar si en el Perú puede ocurrir lo mismo?, ¿será posible implementar esta tecnología en las aulas de gestión pública?, ¿los docentes peruanos se encuentran lo suficientemente preparados para asumir un papel activo en la gestión del conocimiento bajo las características de la sociedad moderna?, y de no ser afirmativa la respuesta a la última pregunta, ¿qué se necesita para lograrlo?

Tantas interrogantes por resolver deberían generar mayor entusiasmo y curiosidad en los docentes del país, no obstante, puede que sea precisamente ése el motivo por el que no se ha tomado la iniciativa en el rubro por indagar acerca de una herramienta novedosa y relativamente complicada para muchos, que algunos de los profesores en nuestra nación posiblemente no se sienten motivados por mejorar su práctica o innovar en sus clases, es probable que exista cierto temor al cambio, pues la pedagogía tradicional ha embargado los salones durante tantos años que se podría suponer que ya muchos se han acostumbrado a ella y, quizás, no se disponen a cuestionar sus resultados.

Dado que la PDI aporta, no solo en cuanto a su uso básico, un conjunto de experiencias y conocimientos que sirven para alcanzar con éxito un objetivo. Como consecuencia se produce una mejora sustancial en los agentes implicados en su autoestima al ser capaces de aumentar sus destrezas tecnológicas (Murado, 2012); es preciso tener claro que tal instrumento "permite la aplicación de nuevas tecnologías en el aula, lo cual torna el aprendizaje más interesante, ameno, motivado e interactivo" (Montoya, 2014, p. 15). Si bien el entusiasmo o motivación que el docente posee al empezar a emplear la PDI no determina el éxito o fracaso final de su incorporación, pues 
constituye "una herramienta aceptada por la generalidad del profesorado por 3 razones: porque es de fácil uso, mejora rápidamente la enseñanza y el aprendizaje, y potencia la creatividad" (SáezLópez, 2011, p. 306), se enfatiza la posibilidad de que los retrasos durante los procesos didácticos "disminuye con la formación y además se amortiza al aumentar el número de sesiones" (Llorente, 2012: 93). No obstante, es crucial evitar en lo posible que el docente, al igual que el discente, se "convierta en esclavo de la tecnología sin saber a ciencia cierta lo que aprende, por qué lo aprende, y lo que, en definitiva, realmente quiere y necesita aprender" (Pando, 2018, p. 481).

En síntesis, si bien la importancia de este trabajo radica en la concientización de docentes en instituciones educativas de todo nivel acerca de capacitarse sobre el buen uso de la tecnología, específicamente de las pizarras digitales interactivas, sobre todo de la relevante influencia de su predisposición actitudinal en el desarrollo de competencias vinculadas a las TIC; se debe tener en cuenta que el contexto actual no resulta-irónicamente-motivador, pues "los docentes conciben la problematización de sus propias prácticas como un hecho puntual, distinto y externo a la enseñanza, que eventualmente podría sumarse a lo que hacen" porque, en ciertos escenarios, "la auto-inspección de lo que se hace no es percibida como una necesidad, menos como un componente esencial en la enseñanza" (Rodríguez-Sosa, 2018, p. 516). Ante el ambiente expuesto, se hace extensivo el aliento a continuar las investigaciones que refieren al uso eficiente de las PDI, de las TIC, de los recursos en general; pero aún más, se exhorta a indagar acerca del papel del docente, no solo como orientador o moderador, sino como un agente tan activo como el estudiante, que aprende y enseña con su apoyo, que admira y es admirado por su labor, que reconoce y es reconocido por su esfuerzo, porque solo así podrá demostrar el orgullo que posee de ser educador, desarrollando sus capacidades al máximo, para que estudiantes y docentes mejoren cada día en beneficio de nuestro país.

\section{Referencias}

Bobbio, N. (1994). Estado, gobierno y sociedad. Por una teoría general de la política. México: Fondo de la Cultura Económica.

Corrales, M. (2015). Selección, elaboración, adaptación y utilización de materiales, medios y recursos didácticos en formación profesional para el empleo. España: Ediciones Paraninfo.

Daccach, J. (s.f.). Tecnologías de la Información y la Comunicación (TIC). Recuperado de: http://www.gestipolis.com/delta/term/TER434.html

Díaz, F. (2009). Las TIC en el aula: TIC y competencias docentes en el siglo XXI. En Carneiro, R., Toscano, J. y Díaz, T. Los desafios de las TIC para el cambio educativo (139-154). Madrid: Editorial Santillana-OEI.

Díaz, I. (2012), El uso de las pizarras interactivas en la educación básica primaria: un estudio en una escuela privada en Costa Rica (Tesis de maestría). Universidad Virtual: Escuela de Graduados en Educación, Costa Rica. Recuperado de: https://repositorio.itesm.mx/handle/11285/619728

Domingo, M. (2011). Pizarra digital interactiva en el aula: uso y valoraciones sobre el aprendizaje. Estudios sobre educación, 20, 99-116. Recuperado de: https://www.unav.edu/publicaciones/revistas/index.php/estudios-sobreeducacion/article/view/4475

Duan, Y. (2010). Teaching interactively with Interactive Whiteboard: Teachers are the key. Networking and Digital Society (ICNDS), 2nd International Conference, 1, $144-147$. Doi: http://dx.doi.org/10.1109/ICNDS.2010.5479608

Cacheiro, M., Dulac, J. \& Gallego, D. (2009). La pizarra digital interactiva como recurso docente. evista Electrónica Teoría de la Educación. Educación y Cultura en la Sociedad de la Información, 10(2), 153-178. Recuperado de: http://espacio.uned.es/fez/view/bibliuned:425-Mlcacheiro-5001

Gatica, N., \& Valdivia, J. (2014). La pizarra digital interactiva y la web 2.0: Ambientes digitales que se sustentan en la teoría del constructivismo social. En Capacitación y gestión del conocimiento a través de la web 2.0 (197-213). Madrid: DYKINSON S, L. 
Llorente, J. (2012). Estudio, evaluación y optimización de los procesos de introducción de las TIC en los centros educativos en formación obligatoria (Tesis doctoral). Universidad Autónoma de Madrid, España. Recuperado de: https://repositorio.uam.es/bitstream/handle/10486/9878/50727_Llorente_Olier_Jose_Ja vier.pdf?sequence $=1$

Luna, E. (2006). El docente presencial: Técnicas de enseñanza para enriquecer su desempeño académico. México: Plaza y Valdés S.A.

Martín, O. (2009). Educación 2.0: Horizontes de la innovación educativa. En Nadal, J. (Ed.), TELOS 78: La escuela digital. Desafíos de la innovación educativa (53-61). Editorial Fundación Telefónica.

Monreal, I. (2013). Uso e integración curricular de la pizarra digital interactiva en el aula de música de primaria. Un estudio de casos en la provincia de Segovia (Tesis doctoral). Universidad de Valladolid, España. Recuperado de: http://uvadoc.uva.es/handle/10324/4228

Montoya, C. (2014). El uso de la pizarra digital en el aula: Experiencia basada en el uso de la PDI de bajo costo. Lima, Perú: Editorial VIRTUAL EDUCA.

Murado, J. (2012). Pizarra digital: Herramienta metodológica integral en el contexto del aula del siglo XXI. España: Ideas Propias.

Niemiec, C., \& Ryan, R. (2009). Autonomy, competence, and relatedness in the classroom applying self-determination theory to educational practice. Theory and Research in Education, 7(2), 133-144. Doi: https://doi.org/10.1177/1477878509104318

Pando, V. (2018). Tendencias didácticas de la educación virtual: Un enfoque interpretativo. $\begin{array}{llll}\text { Propósitos } \quad y \quad \text { representaciones, } & \text { 6(1), } & \text { 463-505. }\end{array}$ http://dx.doi.org/10.20511/pyr2018.v6n1.167

Prensky, M. (2001). Nativos e inmigrantes digitales. Adaptación al castellano por Institución Educativa SEK, España: Distribuidora SEK, S.A. Recuperado de: https://www.marcprensky.com/writing/PrenskyNATIVOS\%20E\%20INMIGRANTES\%20DIGITALES\%20(SEK).pdf

Reeve, J. (2012). A self-determination theory perspective on student engagement. En Handbook of research on student engagement, Nueva York: Editorial Springer.

Rodríguez-Sosa, J., Hernández-Sánchez, K. (2018). Problematización de las prácticas docentes y contextualización de la enseñanza. Propósitos y representaciones, 6(1), 507-541. Doi: http://dx.doi.org/10.20511/pyr2018.v6n1.211

Ryan, R., \& Deci, E. (2000). Intrinsic and extrinsic motivations: classic definitions and new directions. Contemporary Educational Psychology, 25(1), 54-67. Doi: https://doi.org/10.1006/ceps.1999.1020

Sáez-López, J. (2011). Valoración por parte de los docentes del uso y aplicación de la pizarra digital en educación primaria. II Congreso Pizarra Digital. Recuperado de: https://s3.amazonaws.com/academia.edu.documents/45013518/3_II_congreso_PDI.pdf? AWSAccessKeyId=AKIAIWOWYYGZ2Y53UL3A\&Expires=1550183053\&Signature $=\% 2 \mathrm{Fw} 9 \mathrm{VyO} 1 \mathrm{qj} 5 \mathrm{IGHW} \% 2 \mathrm{BHByd} \% 2 \mathrm{FyWf} 9 \mathrm{qJ} \% \% 3 \mathrm{D} \&$ response-contentdisposition=inline \%3B\%20filename\%3DVALORACION_POR_PARTE_DE_LOS_D OCENTES_DE.pdf

Sánchez, E. (2008). Las tecnologías de información y comunicación (TIC) desde una perspectiva social. Revista Educare, 12, 155-162. Recuperado de: http://www.revistas.una.ac.cr/index.php/EDUCARE/article/view/1465 